\title{
Spectral Efficiency Enhancement Through Wavelet Transform Filter Bank for Future Mobile Communications
}

\author{
Ch.Gangadhar, Md. Habibulla
}

\begin{abstract}
In the 5th generation of wireless communications for multiple apps, such as sports, video etc, the large transmission rate of information is the main requirement. To satisfy the high information rates, bandwidth can be increased by using greater frequency bands that is not feasible owing to the restricted frequency spectrum accessibility and the limitation placed on accessible spectrums by Standard. A further way is to effectively use the existing spectrum. OFDM overrides all multiplexing methods in the last century because with Cyclic Prefix (CP) and enhanced Bit Error Rates (BER) the system improves intersymbolic interference (ISI). It offers low sensitivity owing to the intercarrier orthogonality to time synchronization. Bandwidth is lost by $\mathrm{CP}$, in addition to all of these benefits. The lack of orthogonality between pilots that interfere (ICI) is also due to the multipath fading. Discrete Wavelet Transform is used to extract a bandwidth and spectral efficiency improvement and remove $C P$ in turn. Transforming Wavelet (WT) is less sensitive to multipath distortion., so that ICI improves.. We present FBMC's unifying structure, discussion and efficiency assessment in this paper.
\end{abstract}

\section{INTRODUCTION}

Wireless communication today has become a component of our everyday lives. Individuals use various services delivered by service suppliers as required. All wireless systems must deliver extremely high information rates at very high speed, at very low price too. In recent decades, wireless communication has evolved quickly. Reduced noise is necessary in order to incorporate a broad range of applications for example the transmission of high speed information, streaming of video, the internet, and the voice demanding the next generation wireless communication scheme Thus the challenge is the efficient use of the accessible spectrum to accommodate such a enormous amount of customers.Because of its hardness to multipath decay, which is a significant issue in wireless communication, OFDM is the most used method in fourth generation. OFDM also offers elevated transmission rates through divisions of long bit sequences, into tiny bit streams which are orthogonal to each other, at distinct frequencies. In OFDM, equalization Computing difficulty is not necessary. OFDM uses Cyclic (CP) prefix for decreasing inter-symbol interference (ISI) impact, which decreases the spectral efficiency[1]. Transform Wavelet offers multiresolution signal assessment (i.e. time and frequency space). The need for a cyclic prefix (CP) can be eliminated to improve spectral effectiveness by using wavelet filters [2]. Two main findings for potential mobile applications which

Revised Manuscript Received on July 18, 2019.

Ch.Gangadhar, PVP SIDDHARTHA INSTITUTE OF TECHNOLOGY. (Email: gangadharch1111@gmail.com)

Md. Habibulla, PVP SIDDHARTHA INSTITUTE OF TECHNOLOGY.(E-mail: honeyhabeeb@gmail.com) affect the development and modulation of the right multiaccess scheme.

- Flexible allocation of the time frequency to help various user demands and channel characteristics efficiently.

Low delay dispersal, particularly in thick heterogonous networks using beamforming and high carrier frequencies .

For the previous purposes, these two results create FBMC a viable choice for potential portable apps: FBMC can be intended to provide excellent location both at times and at frequencies, so that the available time-frequency resources can be allocated effectively. Secondly, due to its low delay distribution, easy one-tap equalizers are adequate for maximum efficiency.

Recent study has led to cyclical prefixes (CP) using restrictions on frequency use effectively in the areas of the orthogonal frequency division multiplexing scheme (OFDM). The division of broadband into many subchannels of narrowband also results in a large number of side lobes causing a higher loss of energy. OFDM signals steer saturation-near power enhancer because they are characterized by a high peak to mean power ratio (PAPR)[1].

In OFDM Inverse Fourier Transform (IFFT) and the Fast Fourier Transform (FFT), only n-points in the unit circle in the $\mathrm{Z}$ plane remain orthogonal. If the sampling frequency differs little or an interference does not exist in these $\mathrm{N}$ locations the interference extends through subsection. OFDM degrades because of such interference [2].

Slight changes to the time domain of OFDM-based discrete Fourier Transform (DFT) causes inter-symbolic interference (ISI) which is decreased with CP but cost for spectrum efficiency [3]. Based on IFFT OFDM generates elevated side lobes as rectangular windows lead to elevated interference owing to a absence of time domain synchronization. [4]. The wireless multipath fading caused orthogonolty losses in carrier systems because of ISI [5]. OFDM equalization channel results in a substantial bit error rate (BER) and ISI.

OFDM is not flexible and requires IFFT and FFT to be more complicated. The Orthogonal Wavelet Multiplexing Division (OWDM) [ 6 ] offers versatile and less complicated computing with discrete wavelet transformation. OWDM is stronger for haar and daubechies wavelet families. OWDM offers immunity to noise,stronger BER use of bandwidth 


\section{SPECTRAL EFFICIENCY ENHANCEMENT THROUGH WAVELET TRANSFORM FILTER BANK FOR FUTURE MOBILE COMMUNICATIONS}

than OFDM [6]. OFDM utilizes CP to mitigate ISI, reducing spectral efficiency but using Wavelet to perfectly rebuild it can remove $\mathrm{CP}$ and boost spectral efficiency. WPM also offers fewer side lobes that increase the efficiency over OFDM

\section{PROPOSED SYSTEM \& RESULTS}

The orthogonality that decreases Intercarrier Interference (ICI) and ISI along with improved spectral density can be achieved by means of orthogonal filters from wavelete bank transform. So there are no $\mathrm{CP}$ requirements in Wavelet Transform OFDM (WTOFDM) that improve spectrum effectiveness and BER performance.

Based on a good-time frequency localization, multicarrier modulation based upon wavelets is developed that reduces narrowband interference and interference by multichannels With the wavelet packet, the required orthogonal bases can be constructed to modulate the data in multi-carrier, filter bank and wavelet .packet. In order for better performance, a complex wavelet may be used.

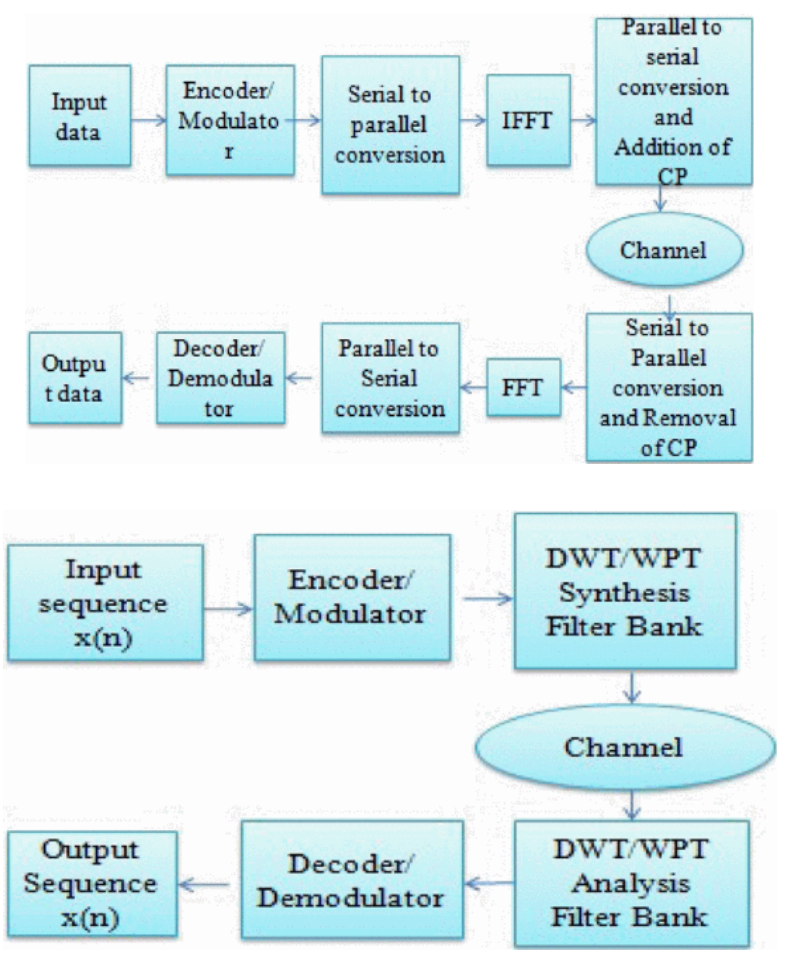

The schematic above shows that the scheme is the same as the OFDM scheme only instead of IFFT or FFT use DWT / WPT synthesis and analysis filter bank extraction. Only when a small signal shift occurs at that time domain, the DFT based OFDM scheme efficient . The quick variation in time or time domain data that tells how the signal works in frequency can be processed simultaneously through time and frequency analysis. Wavelet Transformation can be done using a filter bank can deliver such multi-resolution analyses.

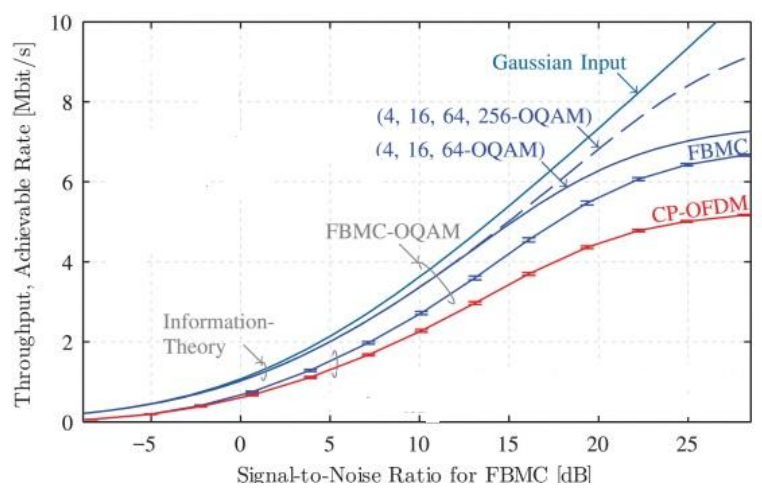

Because of a greater frequency and no CP costs, FBMC has greater performance than OFDM.

\section{CONCLUSIONS AND FUTURE SCOPE}

Wavelet transformation has the main advantage of creating a different bandwidth subcarrier . Denoising wavelet property can also be used for wireless communication. Data compression can be carried out very efficiently via wavelet, thus increasing the data transfer rate and reducing the transmission energy. Due to a greater accessible bandwidth and no overhead CP, FBMC has a greater performance than OFDM.

\section{REFERENCES:}

1. Stridh, R, P. Karlsson, B. Ottersten,"Spatial Characterisation of Measured Indoor Radio MIMO channels at 5 GHz",Proceedings ofNordiskt Radio Symposium / PCC Workshop, 2001.

2. Li, Ye G., J.H. Wintrs, and N.R. Solenberer, "Signal detection for MIMO-OFDM wireless communications", Proceedings ofICC 2001, Vol. 10, pp. 3077-3081,2001.

3. Smulders, P., M. Jevrosimovic, M. Herben , S. Savov, E. Martijn,"State of the art channel models",B4 Deliverable, TUE_WP2_PUB_Oi_vi, TU/e, Eindhoven, April 2002 .

4. H. G. G"ockler and H. Eyssele, "Study of on-board digital FDMdemultiplexing for mobile SCPC satellite communications (part I and II)," Eur. Trans. Telecommun., vol. 3, pp. 7-30, Jan. 1992.

5. I. Djokovic and P. P. Vaidyanathan, "Results on biorthogonal filter banks,"Applicat. Comput. Harmonic Anal., vol. 1, no. 4 pp. 329-343, Sept. 1994.

6. H. G. G"ockler and M. N. Abdulazim, "Tree-structured MIMO FIR filterbanks for flexible frequency reallocation," in Proc. Int. Symp. Image Signal Processing Anal., Istanbul, Turkey, Sept. 2007. 